\title{
Random Number Table
}

National Cancer Institute

\section{Source}

National Cancer Institute. Random Number Table. NCI Thesaurus. Code C142661.

A chart containing numbers, with or without duplication and arranged with no discernable pattern. This table may be used as a statistical tool for selection assignment. 